\title{
Tet Spells in a Parturient Woman with Uncorrected Tetralogy of Fallot Undergoing Cesarean Delivery
}

\author{
Saranya Lertkovit, M.D., Patchareya Nivatpumin, M.D.
}

Department of Anesthesiology, Faculty of Medicine Siriraj Hospital, Mahidol University, Bangkoknoi, Bangkok 10700, Thailand. Received 2 September 2020 • Revised 4 November 2020 • Accepted 18 November 2020 • Published online 8 April 2021

\begin{abstract}
:
Tetralogy of Fallot (TOF) is the most common, cyanotic congenital heart disease. The hemodynamic changes during pregnancy, with uncorrected TOF, result in serious, life-threatening complications for both patient and baby. The authors report on the successful anesthetic management of a 26 -year-old parturient women. The patient was at a gestational age of 33 weeks, with uncorrected TOF. After undergoing a cesarean delivery, she developed intraoperative hypoxia after delivery. We postulated that the patient developed hypoxic Tet spells from hypovolemia, resulting from postpartum bleeding as well as a decrease in her systemic vascular resistance from oxytocin. A multidisciplinary team approach and invasive intraoperative monitoring together with meticulous anesthetic management, were essential for this patient.
\end{abstract}

Keywords: anesthesia, cesarean delivery, hypoxic spell, Tet spells, Tetralogy of Fallot

Contact: Patchareya Nivatpumin, M.D. Department of Anesthesiology, Faculty of Medicine Siriraj Hospital, Mahidol University, Bangkoknoi, Bangkok 10700, Thailand.

E-mail: patchareya.niv@mahidol.ac.th This is an open access article under the CC BY-NC-ND license (http://www.jhsmr.org/index.php/jhsmr/about/editorialPolicies\#openAccessPolicy).

J Health Sci Med Res 2021;39(6):509-515 doi: 10.31584/jhsmr.2021801 www.jhsmr.org 


\section{Introduction}

Tetralogy of Fallot (TOF) is the most common cyanotic congenital heart defect. ${ }^{1}$ TOF is characterized by a ventricular septal defect (VSD); an overriding of the aorta; right ventricular hypertrophy; and right-ventricularoutflow-tract (RVOT) obstruction. Arterial saturation is a direct reflection of the pulmonary blood flow, which depends on the severity of the RVOT obstruction and produces a right-to-left (R-L) shunt. An infundibular spasm, or constriction plays an important role in hypoxic episodes or Tet spells in TOF patients, and directly affects the increments of the catecholamine level and sympathetic tone. There are various, precipitating causes; including, crying, pain, defecation and awakening. ${ }^{2}$ Hypoxia also induces a decrease in systemic vascular resistance (SVR), which further increases the $R-L$ shunt. ${ }^{2}$ Pregnancy with uncorrected TOF involves a maternal mortality rate of 3.0$12.0 \%$, as well as a high rate of perinatal loss. ${ }^{3-5}$ Contained within is the authors report on the successful anesthetic management of intraoperative Tet spells in a woman with uncorrected TOF, who underwent cesarean delivery under general anesthesia.

\section{Case report}

The patient was a 26-year-old, G3P2A0 parturient woman. She was 150 centimeter $(\mathrm{cm})$ in height, with a weight of 42 kilogram (kg) (35 kg before pregnancy), at 33 weeks of pregnancy (As of May, 2020). She had a known underlying disease; Hemoglobin $\mathrm{E}$ trait, with an alpha-1 thalassemia trait. Additionally, she had a prior history of first a vaginal and then a cesarean delivery, both at 28 weeks of gestation. The patient also had a diagnosis of congenital heart disease when she was twelve years old; she had received neither medical, nor surgical treatment for this condition.

She had central and peripheral cyanosis, with a clubbing of her fingers. Her physical examination revealed a systolic ejection murmur, grade IV, at the left upper parasternal border, with a thrill and a loud P2. Electrocardiogram revealed a normal sinus rhythm, and a right ventricular hypertrophy. Her chest radiograph presented cardiomegaly, with interstitial infiltration, in both the lower lung and perihilar regions, and a boot-shaped appearance of the heart (Figure 1). An echocardiogram at 18 weeks of pregnancy showed nonrestrictive, membranous VSD, size $2.5 \mathrm{~cm}$, with bidirectional flow; an overriding aorta; a marked narrowing of the right ventricular outflow tract, and a thickening of the RVOT $(1.1 \mathrm{~cm})$ at $2 \mathrm{~cm}$ from the pulmonic valve; a limit excursion of the pulmonic valve, causing severe subvalvular and valvular pulmonic stenosis (PS); right ventricular systolic pressure (RVSP) of 104 millimeters of mercury $(\mathrm{mmHg})$; no pulmonary hypertension; mild right atium $(R A)$ dilation; right ventricle $(R V)$ hypertrophy and $R V$ dilation; good RV systolic function (Tricuspid annular plane systolic excursion; TAPSE=24 mm); good left ventricular contraction; with a left-ventricular-ejection fraction of $69.0 \%$; and a D-shaped left ventricle (LV), owing to the high RVSP secondary to the subvalvular PS. Her blood-chemistry analysis and coagulation-function tests were both within the normal limits (serum creatinine 0.33 milligram per deciliter (mg/dL), sodium 137 milliEquivalents per liter (mEq $/$ ), potassium $3.7 \mathrm{mEq} /$, chloride $104 \mathrm{mEq} /$, bicarbonate $17 \mathrm{mEq}$ /, Prothrombin time 12.4 seconds and activated Partial Thromboplastin Time 27.4 seconds). Her baseline oxygen saturation was $80.0-85.0 \%$, with a hematocrit of $42.2 \%$. The patient was admitted to the hospital, due to progressive dyspnea and deterioration of her functional status at gestational age (GA) of 25 weeks; for bed rest, oxygen supplementation, and optimized fluid balance between intake (oral fluid, nutrition) in addition to output (urine) during this pregnancy. She received a metoprolol (100 mg) 1/4 tablet twice daily, and an intravenous steroid for promotion of fetal lung maturity. Her blood pressure (BP) was 85-92/50-60 $\mathrm{mmHg}$, and her heart rate was 70-80 beats per minute (bpm). Her functional status was New York Heart Association class III-IV. No hypoxic spell 
occurred in this admission before operation. We assumed the patient had normal preload, by balancing of fluid intake and urine output.

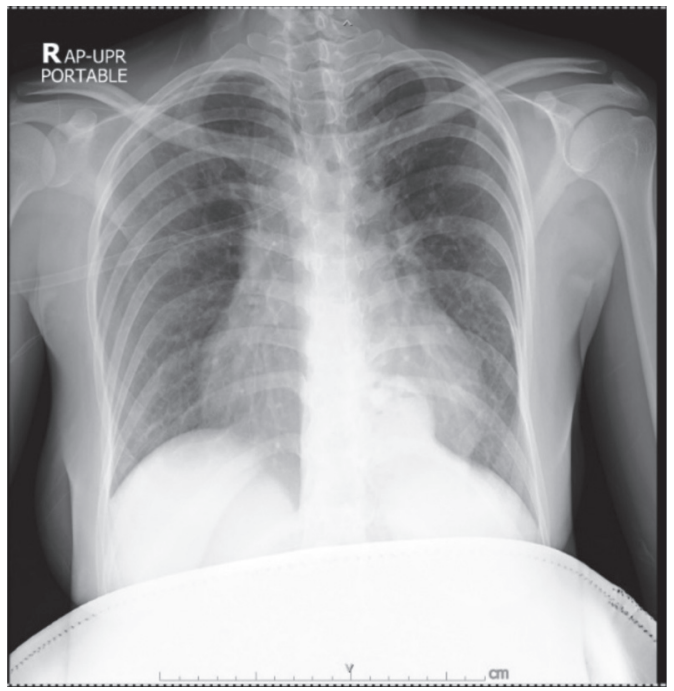

RAP-UPR=right anteroposterior upright portable

Figure 1 Preoperative chest radiograph

The multidisciplinary team, comprised of obstetricians, an obstetric anesthesiologist, a cardiologist and a neonatologist, who all agreed to schedule her for elective cesarean delivery at GA 34 weeks. Nevertheless, she developed premature uterine contraction and dyspnea; therefore, an urgent cesarean delivery was set at GA 33 weeks gestation. We prepared blood components for intraoperative bleeding; comprising of 2 units of packed red cells, 5 units of platelet concentrate and $500 \mathrm{~mL}$ of fresh frozen plasma.

In the operating theatre, her BP was $110 / 60 \mathrm{mmHg}$, her heart rate $90 \mathrm{bpm}$, she had an oxygen saturation $\left(\mathrm{SpO}_{2}\right)$ of $93.0 \%$, and used an oxygen mask with a bag providing $10 \mathrm{~L} / \mathrm{min}$. A left-radial artery catheter, for continuous bloodpressure monitoring, and a central-venous catheter; via the right internal jugular vein under ultrasound guidance, were performed before the induction of general anesthesia under local anesthesia. Bispectral index (BIS) monitoring was used. A 16-gauge intravenous cannula was accessed at her right arm after the induction of anesthesia.

General anesthesia was induced with intravenous ketamine, $75 \mathrm{mg}$ and succinylcholine, $50 \mathrm{mg}$. The patient was intubated without difficulty or desaturation. Anesthesia was maintained with sevoflurane $1.0-2.0 \%$, in $100.0 \%$ oxygen, and cisatracurium at $5 \mathrm{mg}$ was administered. Oxygen saturation was maintained between 85.0 and 95.0\%. After delivery, she was given an infusion of intravenous oxytocin of 30 units in normal saline, 0.5 liter, plus 10 units of an intra-myometrium injection, by the obstetrician, in order to facilitate uterine contractions. No oxytocin intravenously bolus was used for this patient. The sevoflurane was then ceased. Subsequently, dexmedetomidine 0.5 microgram per kilogram $(\mathrm{mcg} / \mathrm{kg})$, per hour was continuously intravenously infused. This was combined with midazolam and fentanyl. Five minutes after delivery, the patient's BP fell to $70 / 40 \mathrm{mmHg}$. Her pulse rate was 90 bpm; her central venous pressure (CVP) declined from 8 to $4 \mathrm{mmHg}$; and her $\mathrm{SpO}_{2}$ decreased to $60.0 \%$. We noticed that the patient had a profuse hemorrhage of approximately 800 milliliter $(\mathrm{mL})$, and a Tet spell was suspected. The aggressive management included volume resuscitation with Lactate Ringer's solution and $500 \mathrm{ml}$ of colloid (Voluven ${ }^{\circledR}$, Fresenius/Hospira, Germany) coupled with increments of intermittent intravenous norepinephrine, for a total of $40 \mathrm{mcg}$. Sodium bicarbonate and ultra-short acting beta-blocker (Esmolol) were available for treatment of this Tet spell. After this, the patient also received one unit of packed red cells $(257 \mathrm{~mL})$. Her blood pressure improved to $100 / 40 \mathrm{mmHg}$ within 15 minutes, her $\mathrm{SpO}_{2}$ increased gradually and was maintained at $>85.0 \%$; and her heart rate was 70-80 bpm. A neonate was delivered with a birthweight of 1,450 gram, with an Apgar score of 8 and 10 at 1 and 5 minutes, respectively. The operation lasted 50 minutes. The patient was not extubated, because of the team's awareness of the oxygenation problem. She was then transferred 
to the cardiac intensive care unit (CCU); there she was successfully extubated, on the fifth hour after her operation. Figure 2 shows the patient's chest $\mathrm{X}$-ray at the fourth hour, postoperatively. She was discharged from the CCU and then from the hospital on postoperative day two and eight, respectively. Her home medications were Metoprolol, iron tablets, calcium carbonate, and Paracetamol. She was scheduled to have a cardiac catheterization and follow-up with the cardiologist. Her baby was admitted into the nursery ward, and placed on an incubator for two days. The baby's problems were neonatal jaundice, due to prematurity and oromotor dysfunction, which were resolved the following week. The total length of the infant's hospital stay was 20 days.

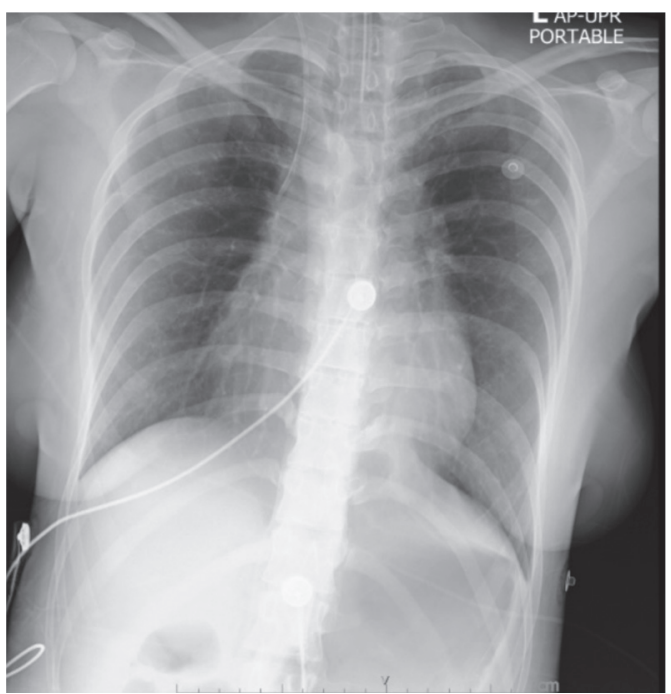

Figure 2 Chest radiograph at $4^{\text {th }}$-hour postoperative

\section{Discussion}

Tet spells in TOF patients can be life-threatening. The mechanism of Tet spells correlates with an increasing severity of infundibular spasms/obstruction, or a decreasing systemic vascular resistance (SVR); resulting in a further increase of R-L shunting of blood (a decrease in the pulmonary blood flow). Consequently, hypoxia develops.
The clinical symptoms of the spells are cyanosis and hyperpnea (an increase in both the rate and the depth of the respiratory pattern). Polycythemia is often related to tissue hypoxia. Patients with polycythemia are associated with an increase in blood viscosity; therefore, they develop vasodilation in order to improve their tissue oxygenation. ${ }^{1}$

The physiological changes during pregnancy include an increase in the prostacyclin and progesterone hormone levels; aggravated TOF symptoms, due to a decrease in SVR, and thus, a worsening, in terms of the R-L shunting of blood. The anesthetic goals for parturients with uncorrected TOF are to provide adequate anesthesia, so as to prevent intraoperative Tet spells by maintaining the preload. This is to avoid tachycardia, an increase in cardiac contractility, and an increase in pulmonary vascular resistance; and to maintain systemic vascular resistance. ${ }^{6}$

The anesthetic techniques of choice for patients with TOF remain controversial. Our patient was an uncorrected one, so decreasing her SVR from regional anesthesia was a major concern, because a neuraxial blockage causes a decrease in the preload and SVR, due to a sympathetic blockage and an increase in the $R-L$ shunt. Alkaya Solmaz et al. ${ }^{7}$ reported a successful application of epidural anesthesia in a woman with uncorrected TOF. Parker and Grange $^{8}$ also reported a successful anesthetic management, with combined low-dose spinal and epidural anesthesia in a 36-weeks parturient woman, with uncorrected TOF. However, our patient had a substantially high RVSP (more than $100 \mathrm{mmHg}$ ) since before her operation. We therefore opted to use general anesthesia, so as to avoid the decreasing SVR effect from regional anesthesia.

General anesthesia provides hemodynamic stability, allowing for manipulation of the shunt fraction with ventilation strategies. Ketamine was chosen as an induction agent in this case upon the effect on her sympathetic nervous system- an increasing cardiac output and a minimal effect on the SVR. ${ }^{2}$ After delivery, sevoflurane was ceased due 
to the team's awareness of its effect on myometrial contractions. The authors chose dexmeditomidine, because it is a highly selective $\alpha-2$ adrenergic agonist that provides sedation, and minimizes the effects of hemodynamic instability. ${ }^{9,10}$ The authors used dexmedetomidine, combined with midazolam and fentanyl, to achieve an adequate depth of anesthesia; guided by BIS monitoring. In obstetrical anesthesia, dexmedetomidine has been use safely during normal labor for sedation, cesarean delivery and shivering prevention, with a low incidence of fetal distress due to high placental retention. ${ }^{11}$ Dexmedetomidine has a low molecular weight, but high protein binding; therefore, transferring this drug through breastmilk is limited. Yoshimura et al. ${ }^{12}$ concluded that perinatal maternal use of dexmedetomidine is unlikely to be harmful to breastfeeding infants. Additionally, dexmedetomidine is a useful adjuvant during general anesthesia for cesarean deliveries, as it attenuates the hemodynamic response to surgical stress and reduces the need for analgesic and anesthetic drugs. ${ }^{13}$ Hence, it was suitable for our patient.

The treatments of a Tet spells include: administering $100 \%$ oxygen, enhancing the preload; by administering $15-30 \mathrm{~mL} / \mathrm{kg}$ of crystalloid solution to increase the heart size and RVOT diameter. Additionally, administering sodium bicarbonate at $1-2 \mathrm{mEq} / \mathrm{kg}$, to treat severe metabolic acidosis. Increasing the SVR and reducing the R-L shunt, via a bolus of a vasopressor. Reducing infundibular spasms, by depressing contractility with a beta-blocker e.g: Propranolol or Esmolol. One must also carefully avoid hyperventilation of the lungs with high airway pressure, since this will further reduce pulmonary blood flow. Then finally, by performing extracorporeal-membrane-oxygenation resuscitation when preparing for a refractory episode, or doing cardiac surgery to relieve the outflow-tract obstruction. This is done by resection of hypertrophied, obstructing muscle bundles and augmenting or enlarging the outflow tract. ${ }^{2}$
Hypovolemia, during cesarean deliveries, frequently occur in patients with uterine atony and postpartum hemorrhaging. Minimal alterations in volume status cause intraoperative hypoxic spells, due to a decrease in the preload, a decrease in the diameter of the RVOT, and a reduction of pulmonary blood flow. In this case, the patient developed Tet spells after delivery, which were derived from acute blood loss. As a result, there was a decrease in the blood flow through her lungs, and she developed tachycardia. This caused an RVOT obstruction. The authors corrected the Tet spell by increasing the intravascular volume with crystalloid and colloid fluids. Although, the blood-transfusion strategies for patients with congenital heart disease are not well-defined ${ }^{14}$, our patient clearly benefited from this treatment. Patients with cyanotic heart disease have high hemoglobin and hematocrit levels, which compensate for the small volume of pulmonary blood flow. We endeavored to keep her hematocrit level close to the preoperative value, so we transfused one unit of packed red cells.

We postulate that another precipitated cause of Tet spell in this patient was a uterotonic agent. Oxytocin, the first-line uterotonic agent, was used in this patient at an incremental dose, via continuous intravenous administration. The concentration of oxytocin infusion was used according to the postpartum hemorrhage management guidelines by the American College of Obstetricians and Gynecologists. ${ }^{15}$ We avoided an intravenous bolus of oxytocin, which caused a precipitous decline in SVR; resulting in decreased blood pressure. ${ }^{16}$ We administered norepinephrine in order to increase the patient's SVR, as phenylephrine was not available in our institute at the time of surgery. We discussed with the obstetrician as to using an additional uterotonic agent, and agreed to use an oxytocin intra-myometrium injection. The second- and third-line uterotonic agents, including ergot alkaloid and prostaglandin, were not used because of their cardiovascular and pulmonary hypertensive effects. $^{16-18}$ 
TOF is a severe type of congenital heart disease that poses substantial risks to both mother and fetus. Anesthetic management requires a good understanding of anatomical defects and physiological adaptations. The medical staff must be aware of the events as well as drugs that affect the magnitude of the R-L shunt. Our coordinated, multidisciplinary care approach led to a successful maternal and neonatal outcome.

\section{Conclusion}

The authors report on the successful anesthetic management of hypoxic 'Tet spells', derived from hypovolemia, due to postpartum bleeding, along with a decrease in the systemic vascular resistance from oxytocin. The aim of our treatment was to increase her pulmonary blood flow, so as to improve oxygenation. Optimum fluid management coupled with judicious administration of anesthetic medications is essential in such cases.

\section{Funding sources}

None

\section{Conflict of interest}

There were no conflicts of interest.

\section{References}

1. Baum VC, De Souza DG. Congenital heart disease in adults. In: Kaplan JA, Augoustides JGT, editors. Kaplan's cardiac anesthesia for cardiac and noncardiac surgery. $7^{\text {th }}$ ed. Philadelphia PA: Elsevier; 2017;p.818-42.

2. Nasr VG, Dinardo JA. Tetralogy of Fallot (TOF). In: Nasr VG, Dinardo JA, editors. The pediatric cardiac anesthesia handbook. Hoboken: John Wiley \& Sons; 2017;p.131-46.

3. Bellapukonda S, Roncall BR, Mund M. Management of a parturient with uncorrected tetralogy of Fallot in shock: an anesthetic challenge. BMJ Case REP 2019;12:e231517.

4. Kaur H, Suri V, Aggarwal N, Chopra S, Vijayvergiya R, Talwar KK. Pregnancy in patients with tetralogy of fallot: outcome and management. World J Pediatr Congenit Heart Surg 2010; 1:170-4.

5. Wang K, Xin J, Wang X, Yu H, Liu X. Pregnancy outcomes among 31 patients with tetralogy of Fallot, a retrospective study. BMC Pregnancy Childbirth [serial on the Internet]. 2019 Dec [cited 2020 May 21]; 19:486. Available from: http:// bmcpregnancychildbirth.biomedcentral.com/articles/10.1186/ s12884-019-2630-y

6. Tawifik MM, Tarbay Al, Abdelkhalek A. Cesarean section in parturients with uncorrected tetralogy of Fallot. Int $\mathrm{J}$ Obstet Anesth 2015;24:191-8.

7. Alkaya Solmaz F, Cuhruk H. The application of epidural anesthesia in pregnant woman with uncorrected tetralogy of Fallot: a case report. Agri 2015;27:215-8.

8. Parker JA, Grange C. Anaesthetic management of a parturient with uncorrected tetralogy of Fallot undergoing caesarean section. Int J Obstet Anesth 2015;24:191-2.

9. Shukry M, Miller JA. Update on dexmedetomidine: use in nonintubated patients requiring sedation for surgical procedures. Ther Clin Risk Manag 2010;6:111-21.

10. Gertler R, Brown HC, Mitchell DH, Silvius EN. Dexmedetomidine: a novel sedative-analgesic agent. Proc (Bayl Univ Med Cent) 2001;14:13-21.

11. Bautista $L$. Dexmedetomidine for every cesarean delivery....maybe not?. Can J Anesth 2019;66:751-4.

12. Yoshimura M, Kunisawa T, Suno M, Sugawara A, Kurosawa A. Intravenous dexmedetomidine for cesarean delivery and its concentration in colostrum. Int J Obstet Anesth 2009;18:403-7.

13. Ibrahim EEA, Amer MM, Ab, Abuelnaga ME, Abd-Elaal W. Dexmedetomidine infusion during caesarean section under general anaesthesia: evaluation of maternal awareness using BIS, maternal and neonatal outcomes. Egypt J Anaesth 2018; 34:145-50.

14. Wilkinson KL, Brunskill SJ, Doree C, Trivella M, Gill R, Murphy MF. Red cell transfusion management for patients undergoing cardiac surgery for congenital heart disease. Cochrane Database Syst Rev 2014,7;CD009752.

15. Committee on Practice Bulletins-Obstetrics. Practice Bulletin No.183: Postpartum Hemorrhage. Obstet Gynecol 2017;130: e168-86.

16. Nivatpumin P. Uterotonic Agents Used in Cesarean Delivery. Thai J Anesthesiol 2019;45:124-31. 
17. Clivatti J, Smith RL, Sermer M, Silversides C, Carvalho JC Cardiac output monitoring during cesarean delivery in a patient with palliated tetralogy of Fallot. Can J Anaesth 2012;59: 1119-24.
18. Arendt KW, Lindley KJ. Obstetric anesthesia management of the patient with cardiac disease. Int J Obstet Anesth 2019;37: 73-85. 